\title{
LASER DIAGNOSTICS
}

DE93 001972

\section{for PICOSECOND e-BEAMS *}

\author{
I. Pogorelsky \\ STI Optronics and Brookhaven National Laboratory \\ I. Ben-Zvi \\ Brookhaven National Laboratory \\ Upton, NY 11973
}

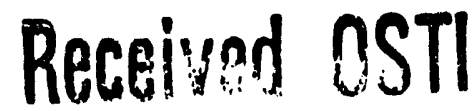

\begin{abstract}
We propose a novel approach to picosecond e-bunch/laser pulse synchronization and spatial alignment based upon refraction and reflection of a laser beam on a plasma column created by relativistic electrons trevelling through a gas or solid opiical material. The technique may be used in laser accelerators and for general subpicosecond e-beam diagnostics.

\section{INTRODUCTION}

A number of laser acceleration proof-of-principle experiments are being initiated or are already under way in several laboratories all over the world. Most of the schemes are based upon tightly focused laser and e-beam interaction on a picosecond time scale. One of the examples is the Inverse Cerenkov Laser Accelerator (ICLA) experiment at the Accelerator Test Facility at Brookhaven National Laboratory where 6-ps e-bunches will be accelerated up to $>10 \mathrm{MeV}$ along $20 \mathrm{~cm}$ by interacting with a 30-ps $\mathrm{CO}_{2}$ laser pulse $^{1}$. The laser beam will be focused by an axicon mirror onto a $200 \mu \mathrm{m}$ diameter (FWHM) e-beam in a hydrogen cell.

Such small time- and space-scale interactions require specific monitoring methods for laser and e-beam alignment and synchronization. A combination of conventional visualizing techniques (phosphor screen, IR TV viewer) allows e-beam/laser spatial alignment to within $\approx 100 \mu \mathrm{m}$. But picosecond e-beam/laser synchronization may be a problem which has not been addressed yet.

We discuss here a method of e-beam monitoring using refraction and total reflection of a laser beam off a plasma column created by a relativistic e-beam propagating through an optically transparent medium. In the following paragraphs we consider refractive index radial gradients created in a medium along the e-beam path, oblique insident laser beam reflection on such gradients, and how this effect may be used for picosecond e-beam diagnostics. Our quantitative estimates are based on real parameters of the ATF $50 \mathrm{MeV}$ e-beam and $10.6 \mu \mathrm{m} \mathrm{CO}$ laser beam propagating in $\mathrm{H}_{2}$ or Ge. The results may be easily extended to other cases of e-beam and laser parameters as well as to various other media.
\end{abstract}

OCT 261992

\section{REFRACTIVE INDEX GRADIENTS IN e-BEAMS}

An e-beam with a radial density profile $N(r, t)$ propagating in any medium or vacuum will create a negative lens due to a refractive index $n$ drop according to the equation

$$
n(r, t)=n_{o}\left(1-N(r, t) / N_{c}\right)^{1 / 2}
$$

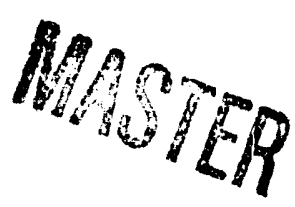

*This work is performed under the auspices 0 " the U.S. Department of EnergY 
which follows from the relations

$$
n^{2}=n_{o}^{2}\left(1-\frac{\omega_{p}^{2}}{\omega^{2}}\right) \text { and } \omega_{p}^{2}=\frac{4 \pi N e^{2}}{m^{*}}
$$

where

$$
\begin{aligned}
\omega_{p} & =\text { plasma frequency; } \\
\omega & =\text { radiation frequency; } \\
\mathrm{N}_{c} & =\text { critical electron concentration when } \omega_{p}=\omega ; \\
\mathrm{m}^{*} & =\text { effective electron mass. }
\end{aligned}
$$

Let us estimate the drop of the refractive index

$$
\Delta n(r, t)=n_{0}-n(r, t),
$$

within the ATF e-beam for the following parameters: electron energy $E_{e}=50 \mathrm{MeV}$, electron bunch charge $Q=0.1 \mathrm{nC}$, pulse width $\tau=6 \mathrm{psec}(\mathrm{FWHM})$, radius $r_{\mathrm{o}}=100 \mu \mathrm{m}$ (FWHM). For the $\mathrm{CO}_{2}$ laser $10.6 \mu \mathrm{m}$ radiation, $\mathrm{N}_{c}=10^{21} \mathrm{~cm}^{-3}$ for $50 \mathrm{MeV}$ electrons with a relativistic factor $\gamma=100$. The total number of electrons in the bunch $\mathrm{Q} / \mathrm{e}=6 \times 10^{8}$. The peak density is

$$
N\left(0, t_{p}\right)=\frac{Q}{e \tau c \pi r_{0}^{2}}=10^{13} \mathrm{~cm}^{-3} .
$$
relation

For the case of $\frac{\omega_{s}^{2}}{\omega^{2}}<1$, the drop of the refractive index $\Delta n$ obeys an approximate

$$
\frac{\Delta n(r, t)}{n_{0}} \approx \frac{1}{2} \frac{\omega_{p}(r, t)^{2}}{\omega^{2}}=\frac{N(r, t)}{2 N_{c}}
$$

and even at the peak relativistic electron concentration, this ratio is very small,

$$
\frac{\Delta n\left(0, t_{p}\right)}{n_{0}} \approx 5 \times 10^{-\theta} \text {. }
$$

However, the secondary electron production along the e-beam path will greatly enhance this effect. First of all, in the case of thermal electrons, the critical electron density is 100 times s.naller, $N_{c}=10^{10} \mathrm{~cm}^{-3}$. The secondary electron density produced at the end of the pulse is

$$
N_{1}(r)=\alpha N\left(r, t_{p}\right) \tau c,
$$

where $\alpha$ is a multiplication factor astimated according to the formula

$$
\alpha=S \rho / E
$$

where

$$
\begin{aligned}
& \mathbf{S}=\text { stopping power } \\
& \rho=\text { medium density; } \\
& \tilde{\mathbf{E}}=\text { average excitation er.2rgy. }
\end{aligned}
$$


Let us consider two examples characteristic of the conditions for the IČLA and typical IR optical materials:

$\underline{\mathrm{H}}_{2}: \mathrm{S}=5.7 \mathrm{MeV} \mathrm{cm}^{2} / \mathrm{g}, \tilde{\mathrm{E}}=19.2 \mathrm{eV},{ }^{2} \rho=8.4 \times 10^{-5} \mathrm{~g} / \mathrm{cm}^{3}$. That gives us $\alpha=25 \mathrm{~cm}^{-1}$ atm $^{-1}$ and

$$
\Delta n=2.2 \times 10^{-6} \mathrm{~atm}^{-1},
$$

along the e-beam axis. Extrapolating this result to other cases of potential interest, we note that $\Delta n$ increases with the atomic number of the gas (e.g. it will be 2 times higher for air and 3 times higher for $\mathrm{Xe}$ ), and it is also proportional to the gas pressure $\mathrm{P}$ and laser frequency $\omega_{L}^{2}$.

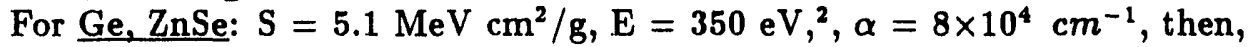

$$
\frac{\Delta n}{n_{0}} \approx 10^{-2} \text {. }
$$

$n_{o}=4$ for $\mathrm{Ge}$ and $n_{o}=2.4$ for $\mathrm{ZnSe}$. This result is valid for relatively thin slabs when multiple scattering may be neglected. The limits imposed by multiple scattering are considered below.

We will see below that such refractive index changes may result in appreciable laser beam distortions in the case of oblique incidence of the laser radiation onto a nonuniform secondary electron plasma column.

\section{LASER BEAM REFLECTION INDUCED BY AN e-BEAM}

From the theory of electromagnetic waves propagation through an isotropic inhomogeneous medium (particularly a plasma) it is known that in the case of oblique incidence total internal reflection will take place from the region within the plasma layer where $n$ drops to the value

$$
n(r)=n_{o} \sin \beta_{o},
$$

where $\beta_{o}=$ angle of incidence. ${ }^{3}$

This effect is responsible for the reflection of radio waves by the ionosphere, as well as of visible laser beams by laser induced plasmas. ${ }^{4}$ We should expect the same effect when a laser beam crosses an e-beam at oblique incidence.

Figure 1 illustrates the effect of total internal reflection expected when a laser beam propagates through a radially inhomogeneous plasma created by an e-beam. In the approximation of geometric optics, that is when

$$
\frac{\lambda_{o} \frac{d n}{d r}}{2 \pi n^{2}}<<1
$$

the trajectory of the laser beam is described by the equation ${ }^{3}$

$$
z=\int \frac{\sin \beta_{o} d r}{\sqrt{n^{2}(r)-\sin ^{2} \beta_{o}}}
$$

Strictly speaking, the geometric optics approximation is not applicable in thevicinity of a "reflection point" where wave equations should be used to calculate the reflection coefficient and phase shift for every particular polarization. But that fact does not appreciably change the condition of total internal reflection and its effects on the beam trajectory. 
At a small angle between the laser and e-beam $\theta_{0}=\frac{\pi}{2}-\beta_{0}$, the condition for total internal reflection will be

$$
\frac{\Delta n}{n_{0}}>\frac{\theta_{0}^{2}}{2} \text { or } \theta_{0}<\sqrt{\frac{N}{N_{e}}} .
$$

In the case of the ATF e-beam, conditions for the total reflection of the $\mathrm{CO}_{2}$ laser beam will be fulfilled in 2 atm $\mathrm{H}_{2}$ when $\theta_{0}<3$ mrad $\approx 10 \mathrm{~min}$, and in Ge when $\theta_{0}<0.15 \mathrm{rad} \approx 10^{\circ}$ (angle measured inside the material).

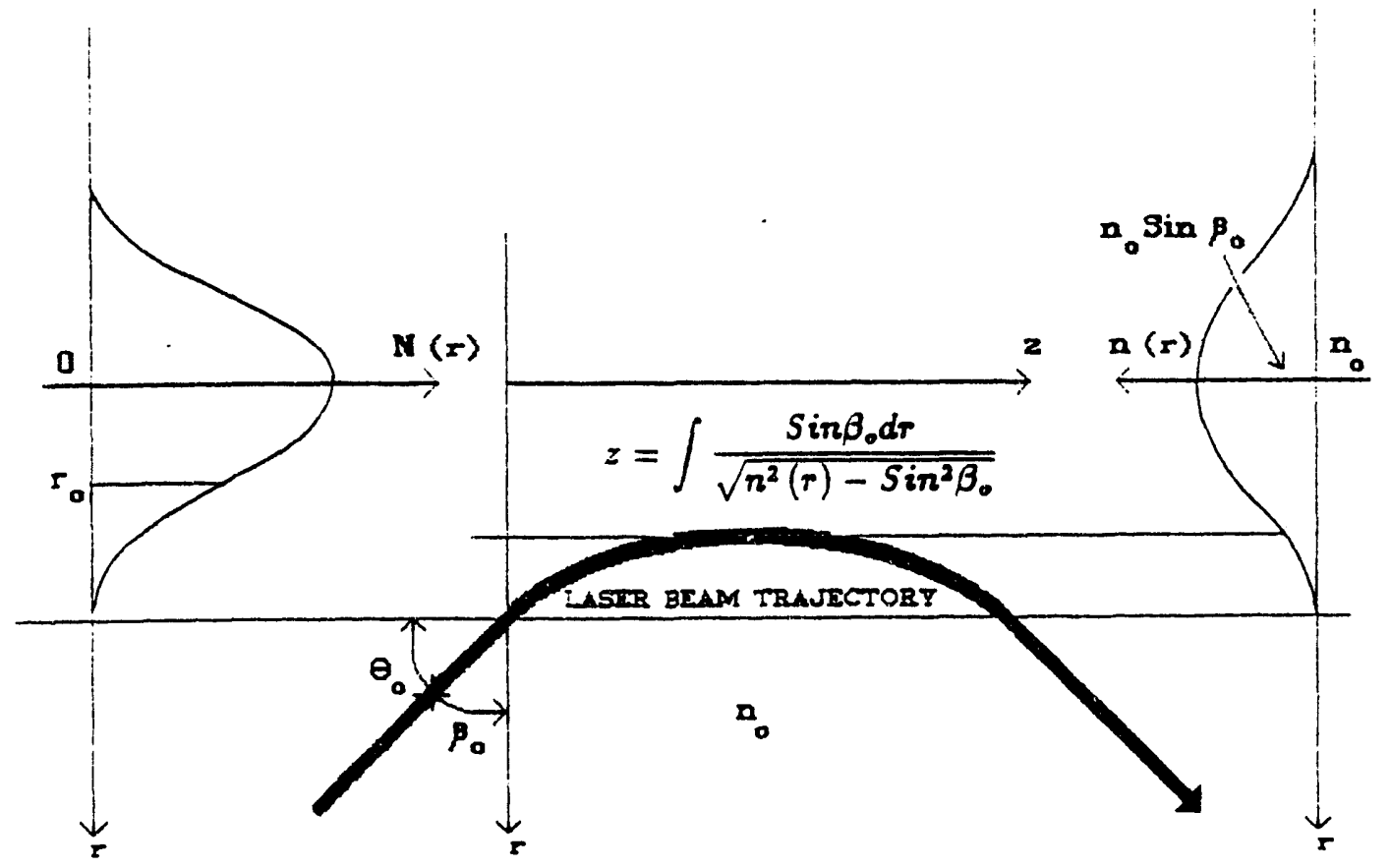

Figure 1: Oblique incidence of electromagnetic wrive on an inhomogeneous plasma. $N(r)$ is the e-beam density and $n(r)$ i $;$, the refrective index.

\section{LASER AND ELECTRON BEAM AIIGNMENT AND SYNCHRONIZATION}

A laser beam reflection, discussed above, may be used for laser and e-beam synchronization within the interaction region. The characteristic time scale for the ricombination and ambipolar diffusion of the secondary electron plasma is $\approx 10^{-8} \mathrm{sec}$ in solids and $\approx 10^{-0} \mathrm{sec}$ in gases. That makes it possible to observe the effect even at relatively long delays of the laser pulse after the passage of the picosecond e-bunch. At that point a precise alignment of the beams may be done. Then laser pulse can be delayed until a reduction and finally disappearance of the effect is observed. This gives the temporal synchronization of the e-beam and laser pulses with the ultimate resolution limited by the width of these pulses.

Methods for implementing this laser beam reflection observation technicue are straightforward and vary according to the optical geometry used to concentrate the laser beam onto the e-beam. For example, a $\mathrm{CO}_{2}$ laser beam with an initial diameter $1 \mathrm{~cm}$, focused by a $\mathrm{f}=1 \mathrm{~m}$ lens, will be confined within a $5 \mathrm{mrad}$ angle to the e-beam 
axis and will be totally reflected by the ATF e-beam in 2 atm of air. Some asymmetry of the initial spatial intensity distribution of the laser beam may be required in order to observe the reflection effect during coaxial illumination. Such an asymmetry may be introduced by a screen blocking a part of the laser beam.

A similar mechanism may be used for alignment and synchronization of an axiconfocused laser beam. In the case of $\theta_{o}=20 \mathrm{mrad}$ (ATF IČLA experiment), $15 \mathrm{~atm}$ Xe will be needed to achive the total reflection condition. As mentioned above, we can increase the secondary electron production orders of magnitude by using dense optical materials. That makes it much more easy to reach the conditions for the total reflection. When considering solid materials for the e-beam diagnostics, we should take into account multiple scattering of the relativistic electrons causing an expansion of the initially filementary e-beam

$$
\Delta r(z)=\frac{2}{3} \frac{E_{S}}{E_{e} \sqrt{L}_{R}} z^{3 / 2}
$$

This follows from the equation: ${ }^{5}$

$$
\Omega_{1 / \mathrm{e}}=\frac{E_{S}}{E_{e}} \sqrt{\frac{z}{L_{R}}}
$$

where

$$
\begin{aligned}
\Omega_{1 / e} & =\text { the rms scattering angle } \\
\mathrm{E}_{S} & =\text { multiple scattering constant }=15 \mathrm{MeV}^{\circ} \\
\mathrm{z} & =\text { distance inside the scatterer } \\
\mathrm{L}_{R} & =\text { radiation length. }
\end{aligned}
$$

Using the tabulated $\mathrm{L}_{R}$ values ${ }^{\theta}$ for solid materials and the proportionality of $\mathrm{L}_{R}$ to $\mathrm{Z}^{2} / \mathrm{A}\left(\mathrm{Z}\right.$ and $\mathrm{A}$ are the atomic number and weight of the scatterer), ${ }^{5}$ we can predict a plasma column expansion in Ge:

$$
\Delta r(z) \approx 0.2 \quad z^{3 / 2}
$$

where $r$ and $z$ are measured in $\mathrm{cm}$.

Due to the drop of the electron density from scattering, the condition for total internal reflection will not be fulfilled at a distance $L$ inside the optical slab unless it satisfies the equation

$$
\theta_{o} L^{3 / 2}<\frac{3}{2} n_{o} \frac{E_{e}}{E_{S}} \sqrt{\frac{\alpha Q L_{R}}{e \pi N_{c}}} \quad\left(\text { at } \Delta r>>r_{o}\right),
$$

where $\theta_{o}$ is measured outside the slab. For the conditions of the ATF ICLA experiment, $\mathrm{L}_{G e}<2 \mathrm{~mm}$.

Radiation damage and color center development caused by the e-beam should be considered when choosing an optical material for the e-beam/laser synchronization probe. 


\section{CONCLUSIONS}

In the present paper we emphasized the prospects of using the proposed method for picosecond laser and electron pulse synchronization having in mind mostly the oncoming laser acceleration experiments at the ATF. But this approach may be used also for timeresolved measurements of picosecond e-bunches in other systems. Using state-of-art IR and visible femtosecond mode-locked lasers, subpicosecond monitoring of the e-bunch time structure and its spatial dynamics may be attained.

\section{REFERENCES}

1. W.D. Kimura, I. Pogorelsky, L.C. Steinhauer, S.C. Tidwell, G.H. Kim, and K.P. Kusche, AIP Proceedings of Advanced Accelerator Concepts Workshop, Port Jefferson, NY, June 14-20, 1992.

2. M.J. Berger and S.M. Seitzer, Stopping Powers and Ranges of Electrons and Positrons (2nd Ed.), National Bureau of Standards, NBSIR 82-2550-A, 1982.

3. V.L. Ginzburg, Propagation of Electromagnetic Waves in Plasma, Gordon and Beach, New York, 1961.

4. H.G. Ahlstrom, Laser-Plasma Interaction, Proceedings of XXXIV Session of the Summer School, Les Houches (France), June 30 - July 25, p.1, 1980.

5. V.L. Highland, Nuclear Instruments and Methods, v.129, p.497, 1975.

6. Particle Data Group, Rev. Mod. Phys., v.45, p.S35, 1973

\section{ACKNOWLEDGMENTS}

Authors wish to thank Wayne Kimura for helpful discussions and enthusiasm to test the proposed method in the course of the upcoming ICLA experiment.

This work was supported by the Department of Energy, Contract No. DE-ACO276CH00016 and No. DE-AC06-83ER40128.

\section{DISCLAIMER}

This report was prepared as an account of work sponsored by an agency of the United States Government. Neither the United States Government nor any agency thereof, nor any of their employees, makes any warranty, express or implied, or assumes any legal liability or responsibility for the accuracy, completeness, or usefulness of any information, apparatus, product, or process disclosed, or represents that its use would not infringe privately owned rights. Reference herein to any specific commercial product, process, or service by trade name, trademark, manufacturer, or otherwise does not necessarily constitute or imply its endorsement, recommendation, or favoring by the United States Government or any agency thereof. The views and opinions of authors expressed herein do not necessarily state or reflect those of the United States Government or any agency thereof. 
DATE

FILMED

$12 / 17 / 92$ 
